\title{
Parameter identification in the mathematical model of glucose and insulin tolerance test - the mathematical markers of diabetes
}

\author{
Marija Heffer ${ }^{1}$, Vedrana Ivić ${ }^{1}$ and Rudolf Scitovski ${ }^{2, *}$ \\ ${ }^{1}$ Faculty of Medicine, J. J. Strossmayer University of Osijek \\ Josipa Huttlera 4, 31000 Osijek, Croatia \\ E-mail:〈marija.heffer@mefos.hr, ,vedrana.ivic@mefos.hr $\rangle$ \\ 2 Department of Mathematics, J. J. Strossmayer University of Osijek \\ Trg Ljudevita Gaja 6, 31000 Osijek, Croatia \\ E-mail:〈scitowsk@mathos.hr〉
}

\begin{abstract}
Glucose tolerance test (GTT) is standard diagnostic procedure that tests the efficiency of blood glucose-lowering hormones (insulin, incretins, leptin). Contrary, insulin tolerance test (ITT) is probing efficiency of blood glucose-rising hormones (glucagon, thyroxine, growth hormone, glucocorticoids, adrenalin, noradrenalin). These two hormone systems together maintain blood glucose levels in a narrow range. Various pathophysiological mechanisms give rise to a reversible condition - prediabetes which then progresses to an irreversible chronic disease - diabetes, both marked with deviation of blood glucose levels outside the set range. In diagnostic purpose, the patient is given glucose load, and blood glucose is measured right before and 2 hours after load. Measurements are more frequent after insulin injection (ITT) or if both tests are performed on experimental animals. In this paper we analyse the mathematical model for GTT and ITT. The obtained model function is an useful tool in describing the dynamics of blood glucose changes.
\end{abstract}

Keywords: glucose tolerance test, insulin tolerance test, mathematical model, parameter identification

Received: April 04, 2020; accepted: June 12, 2020; available online: July 07, 2020

DOI: $10.17535 /$ crorr. 2020.0010

\section{Introduction}

Glucose and fatty acids are the main energy sources for various organs of a multicellular organism [6]. While fatty acids are used during fasting periods, sugar intake leads to glucose burning [21]. Because some organs have no substantial energy store and preferentially use glucose (brain, kidney), even during periods of fasting, blood glucose levels are maintained in a narrow range [35]. The liver is responsible for normoglycemia (a blood glucose concentration between 4.4 and $6.1 \mathrm{~mm} / \mathrm{L}$ in fasting) [3] due to its ability to shift its metabolism in direction of storing or producing glucose which is regulated by two hormones with opposite effect - insulin and glucagon [28]. Generally, the body tolerates hyperglycemia (a blood glucose levels above $6.1 \mathrm{~mm} / \mathrm{L}$ in fasting or above $11.1 \mathrm{~mm} / \mathrm{L} 2$ hours after meal) better than hypoglycemia (a blood glucose concentration below $4.4 \mathrm{~mm} / \mathrm{L}$ ). Therefore, hyperglycemia is physiological after meal and in stress conditions [34, 47], while hypoglycemia causes intense symptoms that can progress to impairment of cognition and seizures [36]. The difference in tolerance of high and low plasma glucose levels explains salient development of diabetes, which has led to epidemic numbers of patients in developed countries. Estimated global number of diabetic patients in 2017 was more than 425 million people while further 352 million suffered from impaired glucose

${ }^{*}$ Corresponding author. 
tolerance (prediabetes) and had high risk of developing diabetes [10]. In the USA alone, as high as 1:3 adults suffer from prediabetes and only $10 \%$ is aware of its condition [11] while $70 \%$ of them will develop diabetes over lifetime [45]. Global risk of prediabetes and diabetes is rising due to epidemic of obesity [15, 29] and worldwide socioeconomic changes [44]. Early diagnosis and treatment of prediabetes could reduce the incidence of diabetes.

Diagnosis of prediabetes and diabetes relies on fasting blood glucose (a blood glucose concentration measured after overnight fasting), plasma glucose concentration 2 hours (2hPG) after load in oral glucose tolerance test (OGTT), and hemoglobin glycation (glucose bound to hemoglobin) [4] - all three are static criteria that do not indicate the origin of the disease, rather they reflect the general rise of glucose in blood. A fully developed clinical presentation of the disease includes insulin resistance (unresponsiveness to insulin) of the liver, adipose tissue, kidney and muscle along with a failure of $\beta$ cells function (insulin secreting cells of pancreatic islets) [17]. Pathophysiological explanation is built on gradual increase of insulin resistance compensated by enhanced insulin secretion by $\beta$ cells. Due to glucose toxicity, compensation works till fasting glucose is around $5.5 \mathrm{mM}[32,33]$. Further increase lead to net loss of pancreatic islets. Work on genetically modified animals fueled discovery of additional mechanisms that contribute to development of hyperglycemia; increased secretion of insulin opposing hormone glucagon, accelerated breakdown of lipids in fat cells, deficiency or resistance to incretins (gastrointestinal tract hormones with augmenting effect on insulin secretion after meal), increased renal glucose reabsorption, and development of central nervous system resistance to leptin (gastrointestinal hormone which promotes satiety) and/or insulin [16]. Recently, altered metabolic state, described as metabolic syndrome, was associated with hyperactive stress response what was adding more on already long list of mechanisms contributing to hyperglycemia [25]. It is very likely that prediabetes is a reversible condition that can be corrected with shortterm targeted therapy directed toward one or few mechanisms. In the absence of unambiguous biomarkers to characterize metabolic disorder, OGTT may serve as an useful orientation. In clinical practice, test is performed in only 2 steps - blood glucose is measured before loading and 2 hours after $75 \mathrm{~g}$ of glucose load [4]. Increased values at either or both steps are considered as a sign of disease. In scientific research conducted on humans or animals, more measurements are made after glucose load, and the blood glucose drop is monitored through 3 or even 4 hours [5]. The number of measurements and the number of experimental animals included in each group made it possible to determine a mathematical model that describes the biological phenomenon at the group level. In the medical literature (see e.g. $[2,13,30]$ ) one can usually find examples of the use of the area under the curve (AUC) and the standard deviation of this surface.

A similar problem exists in performing an insulin tolerance test (ITT) - common test for the stress response. The insulin bolus puts the body in a state of metabolic stress (potentially life-threatening) due to hypoglycemia. Animals with elevated stress response have lower glucose excursions due to opposing effect of stress hormones (glucocorticoids, adrenalin and noradrenalin) [31]. Using AUC as a measure of imbalance can hinter early changes in stress response.

Existing mathematical models that explain progression of diabetes are developed on some of the following assumptions: glycemic excursions as a measure of metabolic instability [24, 42], observation that hyperinsulinemia precedes hyperglycemia [23, 46], opposing effects of insulinglucagon on plasma glucose levels [1], glucose toxicity [18], gradual development of insulin resistance [20], and threshold of glucose-insulin regulatory system [43].

We have chosen to use both tests, GTT and ITT, in order to model mechanisms involved in the maintenance of plasma glucose levels. GTT test is probing mechanisms involved in lowering glucose levels which involve hormones such as insulin, leptin and incretins. On the other hand, ITT is probing mechanisms involved in increasing glucose levels and rely on hormones such as glucagon, glucocorticoids, thyroid hormone, adrenaline and noradrenaline. We assume that a precise description of blood glucose changes provoked by GTT and ITT is a good indicator of the 
development of prediabetes and we propose additional parameters that describe mathematical model in detail. Usefulness of generated parameters should be further experimentally tested.

This paper is organized as follows. In the Section 2, a simple mathematical model of GTT and ITT is introduced. In the Section 3, a corresponding parameter identification problem is considered and in the Section 4 solutions of the corresponding differential equations are analysed. Blood glucose levels in GTT are considered in the Section 5, where basic properties and indicators and typical examples are given. In the Section 6 blood glucose levels in ITT are considered with basic indicators and typical examples. Finally, some conclusions are given in the Section 7.

\section{A simple mathematical model of glucose and insulin tolerance test}

Let us consider the concentration of glucose $G$ in blood and the net normal hormonal concentration $H$ as a cumulative effect of all relevant hormones (for example, insulin decreases $G$, while glucagon and cortisol increases $G$ ). A basic model can be written according to [9] as

$$
\begin{aligned}
\frac{\partial G}{\partial t} & =F_{1}(G, H)+J(t) \\
\frac{\partial H}{\partial t} & =F_{2}(G, H) .
\end{aligned}
$$

The function $J$ is the external rate at which the blood glucose concentration is increased due to adsorption rate. We assume that the quantities $G$ i $H$ attain optimal values $G_{0}$ i $H_{0}$ at a point when the patient arrives to hospital on an empty stomach. Then

$$
F_{1}\left(G_{0}, H_{0}\right)=0 \quad \text { and } \quad F_{2}\left(G_{0}, H_{0}\right)=0 .
$$

Since we are interested in the deviation of $G$ and $H$ from their optimal values, let us introduce the substitution

$$
g=G-G_{0} \quad \text { and } \quad h=H-H_{0}
$$

Then

$$
\begin{aligned}
& \frac{\partial g}{\partial t}=F_{1}\left(G_{0}+g, H_{0}+h\right)+J(t) \\
& \frac{\partial h}{\partial t}=F_{2}\left(G_{0}+g, H_{0}+h\right)
\end{aligned}
$$

By using Taylor's theorem and (3) we obtain

$$
\begin{aligned}
& \frac{\partial g}{\partial t}=\frac{\partial F_{1}\left(G_{0}, H_{0}\right)}{\partial G} g+\frac{\partial F_{1}\left(G_{0}, H_{0}\right)}{\partial H} h+J(t) \\
& \frac{\partial h}{\partial t}=\frac{\partial F_{2}\left(G_{0}, H_{0}\right)}{\partial G} g+\frac{\partial F_{2}\left(G_{0}, H_{0}\right)}{\partial H} h
\end{aligned}
$$

This system allows for signs of constants to be determined a priori, which brings us to the following [9]:

$$
\begin{aligned}
& \frac{\partial g}{\partial t}=-m_{1} g-m_{2} h+J(t) \\
& \frac{\partial h}{\partial t}=-m_{3} h+m_{4} g,
\end{aligned}
$$

where $m_{1}, m_{2}, m_{3}$ and $m_{4}$ are positive constants. 
Since we normally have data about either $G$ or $H$, the system (9)-(10) can be written for that value. Let us show how the same can apply to quantity $H$. We will derive the equation (10) by $t$ and insert (9) into the obtained expression

$$
\begin{aligned}
\frac{\partial^{2} h}{\partial t^{2}} & =-m_{3} \frac{\partial h}{\partial t}+m_{4} \frac{\partial g}{\partial t} \\
& =-m_{3} \frac{\partial h}{\partial t}+m_{4}\left(-m_{1} g-m_{2} h+J(t)\right) .
\end{aligned}
$$

After we replace $m_{4} g$ from (10), we obtain

$$
\frac{\partial^{2} h}{\partial t^{2}}+\left(m_{1}+m_{3}\right) \frac{\partial h}{\partial t}+\left(m_{2} m_{4}+m_{1} m_{3}\right) h=m_{4} J(t)
$$

Since $m_{1}+m_{3}>0$, we mark $\alpha:=\frac{1}{2}\left(m_{1}+m_{3}\right)>0$, and since $m_{2} m_{4}+m_{1} m_{3}>0$, we mark $\omega_{0}^{2}:=m_{2} m_{4}+m_{1} m_{3}>0$ and the equation (11) can be written as

$$
\frac{\partial^{2} h}{\partial t^{2}}+2 \alpha \frac{\partial h}{\partial t}+\omega_{0}^{2} h=m_{4} J(t)
$$

The function $J$ is identical to zero except for the very short time interval in which the glucose load is ingested and can be written by using Dirac $\delta$-function.

Similarly, from (9)-(10) we can obtain

$$
\frac{\partial^{2} g}{\partial t^{2}}+2 \alpha \frac{\partial g}{\partial t}+\omega_{0}^{2} g=m_{3} J(t)+\frac{\partial J}{\partial t}
$$

For simplicity, let us suppose that the $t=0$ is the moment in which the glucose load has been completely ingested. Then, for $t \geq 0$, the functions $g$ and $h$ satisfy homogenous linear differential equation of the second order

$$
y^{\prime \prime}+2 \alpha y^{\prime}+\omega_{0}^{2} y=0, \quad \alpha, \omega_{0}^{2}>0 .
$$

Because of the substitution (4), functions $G$ and $H$ satisfy linear differential equations of the second order

$$
\begin{array}{ll}
G^{\prime \prime}+2 \alpha G^{\prime}+\omega_{0}^{2} G=\omega_{0}^{2} G_{0}, & \alpha, \omega_{0}^{2}>0, \\
H^{\prime \prime}+2 \alpha H^{\prime}+\omega_{0}^{2} H=\omega_{0}^{2} H_{0}, & \alpha, \omega_{0}^{2}>0 .
\end{array}
$$

\section{Parameter identification}

If experimental data $\left(t_{i}, G_{i}\right), i=1, \ldots, m$ are known, where $t_{i}$ are time moments of measuring the concentration of glucose $G_{i}$, we can solve the parameter identification problem

$$
\underset{\alpha>0, \omega_{0}^{2}>0, G_{0}, \mu, \nu \in \mathbb{R}}{\operatorname{argmin}} \Phi\left(\alpha, \omega_{0}^{2}, G_{0}, \mu, \nu\right), \quad \Phi\left(\alpha, \omega_{0}^{2}, \mu, \nu\right)=\sum_{i=1}^{m}\left(G_{i}-\hat{G}\left(t_{i} ; \alpha, \omega_{0}^{2}, G_{0}, \mu, \nu\right)\right)^{2},
$$

where $\hat{G}\left(t_{i} ; \alpha, \omega_{0}^{2}, G_{0}, \mu, \nu\right)$ are the values of the function $\hat{G}$ obtained by solving the Cauchy's problem

$$
G^{\prime \prime}+2 \alpha G^{\prime}+\omega_{0}^{2} G=\omega_{0}^{2} G_{0}, \quad G(0)=\mu, G^{\prime}(0)=\nu .
$$

There are several methods for solving this problem. Let us mention smoothing the data method [41], genetic algorithm [37], etc. In our paper we use Mathematica-module [48] 
NonlinearModelFit []. This way we obtain a good approximation of parameters $\alpha, \omega_{0}^{2}$ and $G_{0}$ in the differential equation (15) and optimal initial condition $G(0)$ and $G^{\prime}(0)$ in the corresponding Cauchy problem.

Similarly, by knowing experimental data $\left(t_{i}, H_{i}\right), i=1, \ldots, m$, we can also estimate parameters $\alpha, \omega_{0}^{2}$ and $H_{0}$ in the differential equation (16) and optimal initial condition $H(0)$ and $H^{\prime}(0)$ in the corresponding Cauchy problem.

\section{The solution of differential equations}

By knowing parameters $\alpha, \omega_{0}^{2}$ and $G_{0}$ in the differential equation (15) and optimal initial condition $G(0)$ and $G^{\prime}(0)$, we are able to write the required function $G$ as the solution to the corresponding Cauchy problem, whereby the crucial role belongs to the corresponding characteristic equation

$$
r^{2}+2 \alpha r+\omega_{0}^{2}=0, \quad r \in \mathbb{C} .
$$

We should make difference between the following cases:

D1: If $\alpha^{2}>\omega_{0}^{2}$, then the roots $r_{1}, r_{2}$ of the characteristic equation are negative and mutually different real numbers denoted in a numerically stable form

$$
r_{1}=-\alpha-\sqrt{\alpha^{2}-\omega_{0}^{2}}, \quad r_{2}=\frac{-\omega_{0}^{2}}{\alpha+\sqrt{\alpha^{2}-\omega_{0}^{2}}},
$$

and the solution of the Cauchy problem for the differential equation (15) is obtained in the form

$$
G(t)=G_{0}+C_{1} e^{r_{1} t}+C_{2} e^{r_{2} t}, \quad C_{1}, C_{2} \in \mathbb{R} .
$$

D2: If $\alpha^{2}=\omega_{0}^{2}$, the characteristic equation has a double negative root $r=-\alpha<0$, and the solution of the Cauchy problem for the differential equation (15) is obtained in the form

$$
G(t)=G_{0}+C_{1} e^{-\alpha t}+C_{2} t e^{-\alpha t}, \quad C_{1}, C_{2} \in \mathbb{R} .
$$

D3: If $\alpha^{2}<\omega_{0}^{2}$, the roots $r_{1}, r_{2}$ of the characteristic equation are conjugate complex numbers $r_{1}=-\alpha-i \omega, r_{2}=-\alpha+i \omega, \omega=\sqrt{\omega_{0}^{2}-\alpha^{2}}>0$, and the solution of the Cauchy problem for the differential equation (15) is obtaied in the form

$$
G(t)=G_{0}+e^{-\alpha t}\left(C_{1} \cos \omega t+C_{2} \sin \omega t\right), \quad C_{1}, C_{2} \in \mathbb{R} .
$$

By introducing the corresponding integration constants $A=\sqrt{C_{1}^{2}+C_{2}^{2}}, \delta=\arctan \frac{C_{2}}{C_{1}}$ instead of $C_{1}$ i $C_{2}$, the solution of the Cauchy problem for the differential equation can be written in the form

$$
G(t ; A, \alpha, \omega, \delta)=G_{0}+A e^{-\alpha t} \cos (\omega t-\delta) .
$$

Remark 1. Since in case [D1] for roots (20) of the characteristic equation there holds

$$
r_{1}+r_{2}=-2 \alpha, \quad r_{2}-r_{1}=2 \sqrt{\alpha^{2}-\omega_{0}^{2}},
$$

the corresponding Wronsky's determinant

$$
W\left[e^{r_{1} t}, e^{r_{2} t}\right]=e^{\left(r_{1}+r_{2}\right) t}\left(r_{2}-r_{1}\right)=2 e^{-2 \alpha t} \sqrt{\alpha^{2}-\omega_{0}^{2}}
$$

can be a very small number, which can lead to a numerically unstable solution (21). Note also that, in practical investigations, the case [D2] almost never arises. Therefore, this case is not considered in the rest of the paper. 
The function $G$ of the form (21) or (23), obtained by solving the corresponding Cauchy problem, can be corrected by solving the corresponding nonlinear least squares problem $[19,26]$ for the function $F_{1}$ given by (24) (in case $\alpha^{2}>\omega_{0}^{2}$ ), i.e. for the function $F_{3}$ given by (25) (in case $\left.\alpha^{2}<\omega_{0}^{2}\right)$ :

$$
\begin{aligned}
& F_{1}\left(G_{0}, C_{1}, C_{2}, r_{1}, r_{2}\right)=\sum_{i=1}^{m}\left(G_{0}+C_{1} e^{r_{1} t_{i}}+C_{2} e^{r_{2} t_{i}}-G_{i}\right)^{2} \\
& F_{3}\left(G_{0}, \alpha, \omega, \delta\right)=\sum_{i=1}^{m}\left(G_{0}+A e^{-\alpha t_{i}} \cos \left(\omega t_{i}-\delta\right)-G_{i}\right)^{2} .
\end{aligned}
$$

\section{Blood glucose levels in the glucose tolerance test}

In the time $t=0$ a certain amount of glucose is either injected into the patient's blood or it was administered orally and blood glucose is monitored for the next 4 hours.

\subsection{The characteristics of the solution}

Since $\alpha, \omega_{0}^{2}>0$, for the obtained function $G$ in both previously mentioned cases there holds

$$
\lim _{t \rightarrow+\infty} G(t)=G_{0} .
$$

The number $G_{0}$ represents glucose concentration after a longer time interval upon glucose load - conveniently, the time interval could be set to 120 minutes - a time point that is interesting for the diagnosis of diabetes.

Also, in order to study the behavior of glucose concentration with certain patients, we will use the following characteristics of the obtained function $G$ :

1. Parameter $\alpha$ determines the rate of oscillation amplitude decline and ensures a gradual decrease in blood glucose to $G_{0}$ level;

2. In the case of conjugate complex roots of the characteristic equation $\left(\alpha^{2}<\omega_{0}^{2}\right)$ we can also determine the basic period $\left[T=\frac{2 \pi}{\omega}\right]$ of function $G$;

3. Glucose concentration at the beginning of the experiment $G(0)$ in clinical or laboratory setting equal to fasting glucose;

4. The initial speed of glucose increase after the initialization $G^{\prime}(0)$ - the reaction speed of glucose lowering mechanisms.

5. Maximal glucose concentration $G_{\max }$ or maximal hyperglycaemia achieved in individual testing reflecting the sensitivity of glucose lowering mechanisms to recognize glucose rise. Also, the moment $t_{\max }$ (in minutes) in which the maximum glucose concentration is obtained is important - the reaction time of glucose lowering mechanisms. On the graph of the function $G$, this is the point $M=\left(t_{\max }, G_{\max }\right)$.

6. Maximal speed of glucose concentration decrease $G^{\prime}\left(t_{I}\right)$ is attained at the moment $t_{I}$ (in minutes), where $I=\left(t_{I}, G\left(t_{I}\right)\right)$ is the inflection point of the function $G$. Note also that $G^{\prime}\left(t_{I}\right)$ is a negative number because it represents the decrease of concentration. $G\left(t_{I}\right)$ and $t_{I}$ are potential values describing the incretin effect and indicating the difference between impairment of glucose tolerance and impairment of fasting glucose.

7. The area under the curve AUC $=\int_{0}^{4} G(t) d t$ represents the cumulative glucose load in a tested period of time. 
Example 1. (Normoglycemia) For $\alpha=4.5$ and $\omega_{0}^{2}=25$, the parameter $\omega=\sqrt{\omega_{0}^{2}-\alpha^{2}}=$ $2.179>0$ is determined. Because $\alpha^{2}<\omega_{0}^{2}$, with $G_{0}=100$ and initial conditions: $G(0)=70$, $G^{\prime}(0)=4000$, the mathematical model (15) determines the model-function of the form (23)

$$
G_{N}(t)=100+1773.64 e^{-4.5 t} \cos (2.179 t-1.554)
$$

The graph of this model-function is shown on Figure 1 and their properties are presented in Table 1.

Example 2. (Prediabetes) For $\alpha=2.5$ and $\omega_{0}^{2}=8$, the parameter $\omega=\sqrt{\omega_{0}^{2}-\alpha^{2}}=$ $1.323>0$ is determined. Because $\alpha^{2}<\omega_{0}^{2}$, with $G_{0}=150$ and initial conditions: $G(0)=100$, $G^{\prime}(0)=2500$, the mathematical model (15) determines the model-function of the form (23)

$$
G_{P}(t)=150+1796.03 e^{-2.5 t} \cos (1.323 t-1.554) .
$$

The graph of this model-function is shown on Figure 2 and their properties are presented in Table 2.

Example 3. (Diabetes) For $\alpha=1.5$ and $\omega_{0}^{2}=3.3$, the parameter $\omega=\sqrt{\omega_{0}^{2}-\alpha^{2}}=1.0247$ is determined. Because $\alpha^{2}<\omega_{0}^{2}$, with $G_{0}=250$ and initial conditions: $G(0)=100, G^{\prime}(0)=1800$, the mathematical model (15) determines the model-function of the form (23)

$$
G_{D}(t)=250+1544.34 e^{-1.5 t} \cos (1.025 t-1.473) .
$$

The graph of this model-function is shown on Figure 1 and their properties are presented in Table 1.

\begin{tabular}{|lcccccccccr|}
\hline Properties & $\alpha$ & $T$ & $G(0)$ & $G_{0}$ & $G^{\prime}(0)$ & $G_{\max }$ & $t_{\max }$ & $G^{\prime}\left(t_{I}\right)$ & $t_{I}$ & AUC \\
\hline \hline Normoglycemia & 4.5 & 2.9 & 70 & 100 & 4000 & 394.2 & 12.9 & -579.7 & 24 & 549.2 \\
Prediabetes & 2.5 & 4.7 & 100 & 150 & 2500 & 467.7 & 23.3 & -358.2 & 48 & 881.3 \\
Diabetes & 1.5 & 6.1 & 100 & 250 & 1800 & 564.2 & 40.8 & -237.4 & 78 & 1411.2 \\
\hline
\end{tabular}

Table 1: Properties of GTT model-functions. Progression toward diabetes is accompanied by increase in $T, G_{\max }, t_{\max }$ and $t_{I}$ (T measured in hours, $t_{\max }$ and $t_{I}$ in minutes) as well as lowering of $\alpha, G^{\prime}(0)$ and $G^{\prime}\left(t_{I}\right)$

The mathematical model reveals that progression toward diabetes is accompanied by rise in $T, G_{\max }, t_{\max }$ and $t_{I}$ as well as lowering of $\alpha, G^{\prime}(0)$ and $G^{\prime}\left(t_{I}\right)$. Note that the glucose concentration in normoglycemia decreases the fastest and the decrease happens in shorter time. In diabetes, the glucose concentration decreases the slowest and the decrease happens in longer time. Progression from prediabetes to diabetes is characterized by previously described rise in parameters $G(0)$ and $G_{0}$, both relevant for clinical practice. Also, the accompanying rise in AUC is well described in human and animal studies of diabetes. What is not yet described are parameters $\alpha, T, G_{\max }, t_{\max }, t_{I}, G^{\prime}(0)$ and $G^{\prime}\left(t_{I}\right)$. Further experimental studies are needed to determine whether any of the newly identified parameters is a more sensitive predictor of the onset of diabetes than existing biomarkers.

\section{Blood glucose levels in the insulin tolerance test}

At the time point $t=0,0.1-0.15 \mathrm{U} / \mathrm{kg}$ of insulin is injected into the patient's blood and the level of glucose in blood is observed within the next 4 hours. 


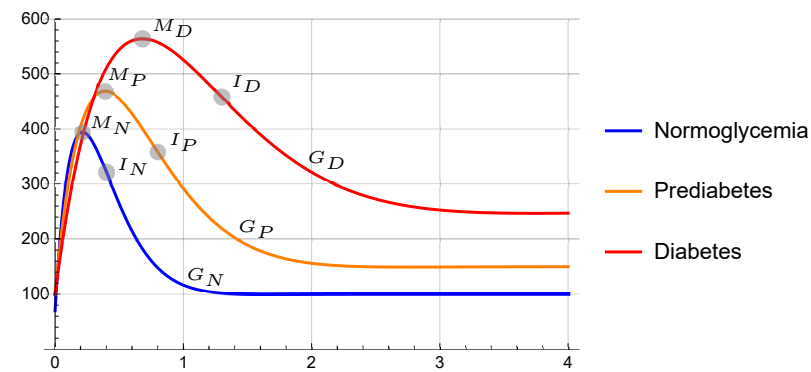

Figure 1: GTT model-functions in the case of normoglycemia $M_{N}=(13,394), I_{N}=(24,321)$; prediabetes $M_{P}=(23,468), I_{P}=(48,358)$ and diabetes $M_{D}=(41,564), I_{D}=(78,457)$ abscissa of each point is in minutes.

Similarly, by knowing experimental data $\left(t_{i}, G_{i}\right), i=1, \ldots, m$, we can solve the corresponding parameter identification problem (17), obtain the parameter values $\alpha, \omega_{0}^{2}$ and $G_{0}$ and estimate the initial conditions $G(0)$ and $G^{\prime}(0)$.

The solution of the Cauchy problem for differential equation (15) with obtained initial conditions depends on the roots of the corresponding characteristic equation (19) and we should also differentiate between the three cases previously mentioned.

\subsection{The characteristics of the solution}

Since $\alpha, \omega_{0}^{2}>0$, for solving the equation (15) in all the previously mentioned cases there holds

$$
\lim _{t \rightarrow+\infty} G(t)=G_{0}
$$

The number $G_{0}$ represents glucose concentration after a longer time interval upon insulin injection - in experimental protocol glucose concentration is conventionally taken up to 60,90 or 120 minutes, but in clinical setting, the last measurement is done after 120 minutes - same as in the case of GTT.

Also, in order to study the behavior of glucose concentration, we will use the following characteristics of the obtained function $G$ :

1. Parameter $\alpha$ determines the speed of oscillation amplitude decrease and provides a trend of glucose increase till the level $G_{0}$;

2. In the case of conjugate complex roots of the characteristic equation, we can also determine the basic period $T=\frac{2 \pi}{\omega}$ of function $G$ (in hours);

3. Glucose concentration $G(0)$ at the beginning of the experiment;

4. The initial speed of glucose decrease $G^{\prime}(0)$ after the initialization of testing (negative number);

5. The minimal glucose concentration $G\left(t_{\min }\right)$ and the moment $t_{\min }$ (in minutes) in which the minimal glucose concentration is achieved;

6. The maximal speed of glucose concentration increase $G^{\prime}\left(t_{I}\right)$ and the moment $t_{I}$ (in minutes) in which the maximal speed of glucose concentration is achieved;

7. The area under curve $\mathrm{AUC}=\int_{0}^{4} G(t) d t$ shows cumulative glucose load in the tested period of time. 
Example 4. (Normal stress response) For $\alpha=0.5$ and $\omega_{0}^{2}=1.5$, the parameter $\omega=$ $\sqrt{\omega_{0}^{2}-\alpha^{2}}=1.12>0$ is determined. Because $\alpha^{2}<\omega_{0}^{2}$, with $G_{0}=80$ and initial conditions: $G(0)=100, G^{\prime}(0)=-100$, the mathematical model (15) determines the model-function of the form (23)

$$
G_{N}(t)=80+82.95 e^{-0.5 t} \cos (1.12 t-1.33) .
$$

The graph of this model-function is shown on Figure 2 and their properties are presented in Table 2.

Example 5. (Enhanced stress response) For $\alpha=0.8$ and $\omega_{0}^{2}=1.5$, the parameter $\omega=$ $\sqrt{\omega_{0}^{2}-\alpha^{2}}=0.93>0$ is determined. Because $\alpha^{2}<\omega_{0}^{2}$, with $G_{0}=120$ and initial conditions: $G(0)=100, G^{\prime}(0)=-100$, the mathematical model (15) determines the model-function of the form (23)

$$
G_{E}(t)=120+126.68 e^{-0.8 t} \cos (0.927 t+1.412) .
$$

The graph of this model-function is shown on Figure 2 and their properties are presented in

\begin{tabular}{|c|c|c|c|c|c|c|c|c|c|c|}
\hline Prop & $\alpha$ & $T$ & $G(0)$ & $G_{0}$ & $G^{\prime}(0)$ & $G_{\min }$ & $t_{\text {min }}$ & $G^{\prime}\left(t_{I}\right)$ & $t_{I}$ & AUC \\
\hline $\begin{array}{l}\text { Norn } \\
\text { respo }\end{array}$ & 0.5 & 5.6 & 10 & 80 & -100 & 4 & 74.8 & 9.7 & 138 & 586.2 \\
\hline $\begin{array}{l}\text { Enhance } \\
\text { response }\end{array}$ & 0.8 & 6.8 & 100 & 120 & -100 & 67.6 & 45.3 & 30.6 & 102 & 872.1 \\
\hline
\end{tabular}
Table 2.

Table 2: Properties of ITT model-functions. Newly identified parameters $\alpha, T, G_{\min }, t_{\min }$ and $t_{I}$ are potential markers of the enhanced stress response

(T measured in hours, $t_{\text {min }}$ and $t_{I}$ in minutes)

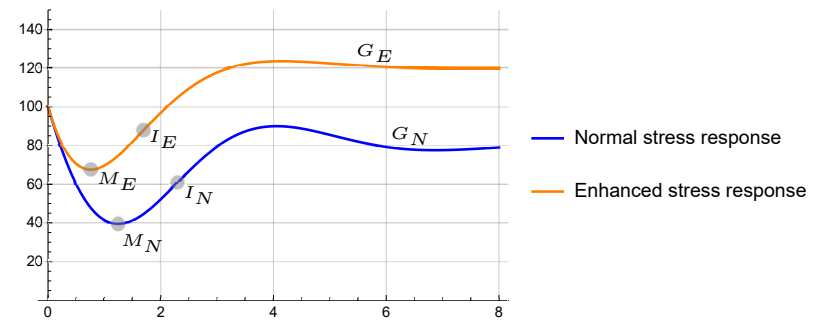

Figure 2: ITT model-functions in the case of normal $M_{N}=(75,39), I_{N}=(138,61)$ and enhanced stress response $M_{E}=(45,68), I_{E}=(102,88)$ - abscissa of each point is in minutes

Note that the speed of glucose level increase in both cases is around 30, but, with normal stress response, the moment of maximal increase is slightly prolonged. On the other hand, parameters $\alpha, T, G_{0}$ and $G_{m i n}$ are increased in the enhanced stress response, while $t_{\text {min }}$, $G^{\prime}\left(t_{I}\right)$ and $t_{I}$ are decreased. Experimental studies will show whether some of them are good biomarkers of changes in a stress response.

\section{Conclusions}

Using hypothetical data and real-life examples of GTT and ITT curves from the literature $[8,14,22,40]$, we have developed a mathematical model that describes changes in blood glucose concentrations challenged by glucose load or insulin bolus. The following indicators describe 
characteristics of the mathematical model based on the set of data: parameter $\alpha$, basic period $T$, glucose concentration at the beginning of the experiment $G(0)$, glucose concentration after a longer time interval upon glucose load $G_{0}$, the initial speed of glucose increase/decrease after the initialization $G^{\prime}(0)$, maximum/minimum glucose concentration $G_{\max } / G_{\min }$ and the corresponding time-moment, maximal speed of glucose concentration decrease/increase $G^{\prime}\left(t_{I}\right)$ and the corresponding time-moment and the area under curve AUC. The indicators $G(0), G_{0}$ and AUC correspond to commonly used measures of risk for prediabetes and diabetes in humans or experimental animals (fasting glucose, $2 \mathrm{hPG}$ and AUC under the GTT or ITT curve), but plotted or extracted from the model. The indicators $G_{\max } / G_{\min }$ and the corresponding timemoment describe glucose excursion in one cycle. The glucose excursion is recognized as a cardiovascular risk [38] which should be attenuated by pharmacological intervention [27, 39]. There are two additional parameters: $G^{\prime}(0)$, which describes the rate of increase/decrease to $G_{\max } / G_{\min }$, and $G^{\prime}\left(t_{I}\right)$, which describes the rate of decrease/increase towards $G_{0}$ at the point $\left(t_{I}, G\left(t_{I}\right)\right)$. We hypothesize that the pharmacological intervention can be better described by the previously mentioned four parameters instead just by AUC, to which the fifth parameter $t_{I}$ can be added.

The basic period $T$ and parameter $\alpha$ have not been used so far to describe changes in blood glucose concentration. Given that glucose-regulating hormones are secreted in cycles, we think it is correct to present changes in blood glucose concentration by a function that stabilizes around its asymptote. The parameter $\alpha$ determines the "quenching rate" around the asymptote. In practical terms, $T$ and $\alpha$ can be seen as indicators of metabolic flexibility. Given that rapidly extinguished functions have higher values of $\alpha$, it is assumed that $\alpha$ is smaller in state with pathological changes especially in the case of GTT test. Let's say that $\alpha$ is higher for healthy young people than for old people. It is to be expected that after a metabolic challenge a healthy young individual quickly returns to baseline, i.e. all fluctuations are within a smaller range. The opposite is true for indicator $T$ - an increase in its value is associated with a greater likelihood of pathological change.

This model was built thanks to a standardized clinical protocol for testing glucose metabolism. Glucose load is an idealized meal without the admixture of other categories of foods - fats, proteins and fibers - and it is not common outside the diagnostic frame. If mixed meals were used in the test, the glucose excursions would be much less [12]. Also, both tests, GTT and ITT, are performed after overnight fasting which further contributes to their standardization - i.e. glucose is readily absorbed in digestive tract after oral glucose administration and gluconeogenesis (production of glucose by liver) is undisturbed by digestion after insulin bolus. Mathematical models that seek to monitor changes in blood glucose concentrations after mixed meal tolerance test [7] have to take into account interference from fat and protein metabolism and rather chaotic circumstances of digestion rate. We assume that the basic mathematical model for monitoring glucose metabolism in the future will be upgraded with models for at least these two additional metabolisms.

A potential weakness of our mathematical model is that we did not recognize which parameter would describe the variability among the experimentally obtained real data which could be a substitute for statistical significance. Nevertheless, we hypothesize that this model could facilitate the comparison of the results from different studies, especially those concerning drug efficacy.

\section{Acknowledgements}

This work was supported by the Croatian Science Foundation through research projects grants IP-2014-09-2324 and IP-2016-06-6545 and University of Osijek grant INGI-2015-35.

The author would like to thank the referees and the journal editors for their careful reading of the manuscript and insightful comments that helped us to improve the paper. Especially, we 
would like to thank Mrs. Katarina Moržan for significantly improving English of this paper.

\section{References}

[1] Adams, C. L. and Lasseigne. D. G. (2018). An extensible mathematical model of glucose metabolism. Part I: the basic glucose-insulin-glucagon model, basal conditions and basic dynamics. Letters in Biomathematics, 5(1), 70-90. doi: 10.1080/23737867.2018.1429332

[2] Allison, D. B., Paultre, F., Maggio, C., Mezzitis, N. and Pi-Sunyer, F. X. (1995). The use of areas under curves in diabetes research. Diabetes Care, 18(2), 245-250. doi: 10.2337/diacare.18.2.245

[3] American Diabetes Association (2000). Screening for type 2 diabetes. Clinical Diabetes, 18(2), http://journal.diabetes.org/clinicaldiabetes/v18n22000/pg69.htm

[4] American Diabetes Association (2010). Diagnosis and classification of diabetes mellitus. Diabetes Care, 33(S1), 62-69. doi: 10.2337/dc10-s062

[5] Ayala, J. E., Samuel, V. T., Morton, G. J., Obici, S. amd Croniger, C. M., Shulman, G. I., Wasserman, D. H. and McGuinness, O. P. (2010). Standard operating procedures for describing and performing metabolic tests of glucose homeostasis in mice. Disease Models and Mechanisms, 3(9-10), 525-534. doi: 10.1242/dmm.006239

[6] Berg, H. M., Tymoczko, J. L., Stryer, L. and Clarke, N. D. (2003). Biochemistry. New York: Freeman.

[7] Besser, R. E. J., Shields, B. M., Casas, R., Hattersley, A. T. and Ludvigsson, J. (2013). Lessons from the mixed-meal tolerance test: Use of 90-minute and fasting $\mathrm{C}$-peptide in pediatric diabetes. Diabetes Care, 36(2), 195-201. doi: 10.2337/dc12-0836

[8] Bowe, J. E., Franklin, Z. J., Hauge-Evans, A. C., King, A. J., Persaud, S. J. and Jones, P. M. (2014). Metabolic phenotyping guidelines: Assessing glucose homeostasis in rodent models. Journal of Endocrinology, 222(G3), 13-25. doi: 10.1530/joe-14-0182

[9] Braun, M. (1983). Differential equations and their applications: An introduction to applied mathematics. In Applied Mathematics book series, 11. New York: Springer. doi: 10.1007/978-1-46124360-1

[10] Centers for Disease Control and Prevention, (2017). Diabetes atlas. International Diabetes Federation. http://www.diabetesatlas.org.

[11] Centers for Disease Control and Prevention, (2017). National diabetes statistics report. Atlanta: U. S. Department of Health and Human Services. https://www.cdc.gov/diabetes/data/ statistics/statistics-report.html.

[12] Charles, M. A., Hofeldt, F., Shackelford, A., Waldeck, H., Dodson, L. E., Bunker, D., Coggins, J. T. and Eichner, H. (1981). Comparison of oral glucose tolerance tests and mixed meals in patients with apparent idiopathic postabsorptive hypoglycemia: Absence of hypoglycemia after meals. Diabetes, 30(6), 465-470. doi: 10.2337/diab.30.6.465

[13] Cheng, K. C., Li, Y. and Cheng, J. T. (2018). The areas under curves used in diabetes research: Update view. Integr Obesity Diabetes, 4(3), 1-2. doi: 10.15761/iod.1000212

[14] Cheng, X., Yang, N., Li, Y., Sun, G., Qiu, L., Xu, L., Ping, L., Li, W. and Zhang, H. (2019). The shape of the glucose response curve during an oral glucose tolerance test heralds $\beta$-cell function in a large chinese population. BMC Endocrine Disorders, 19(1), 1-11. doi: 10.1186/s12902-019-0446-4

[15] Chobot, A., Górowska-Kowolik, K., Sokolowska, M. and Jarosz-Chobot, P. (2018). Obesity and diabetes-not only a simple link between two epidemics. Diabetes/Metabolism Research and Reviews, 34(7), e3042(1-9). doi: 10.1002/dmrr.3042

[16] De Fronzo, R. A. (2009). From the triumvirate to the ominous octet: A new paradigm for the treatment of type 2 diabetes mellitus. Diabetes, 58(4), 773-795. doi: 10.2337/db09-9028

[17] De Fronzo, R. A., Ferrannini, E., Groop, L., Henry, R. R., Herman, W. H., Holst, J. J., Hu, F. B.,Kahn, C. R., Raz, I., Shulman, G. I., Simonson, D. C., Testa, M. A. and Weiss, R. (2015). Type 2 diabetes mellitus. Nature Reviews Disease Primers, 1(1). doi: 10.1038/nrdp.2015.19

[18] De Gaetano, A., Hardy, T., Beck, B., Abu-Raddad, E., Palumbo, P., Bue-Valleskey, J. and Porksen, N. (2008). Mathematical models of diabetes progression. American Journal of PhysiologyEndocrinology and Metabolism, 295(6), E1462-E1479. doi: 10.1152/ajpendo.90444.2008

[19] Dennis, J. E. and Schnabel, R. B. (1996). Numerical methods for unconstrained optimization and nonlinear equations. Philadelphia: SIAM (Society for Industrial and Applied Mathematics). doi: 
$10.1137 / 1.9781611971200$

[20] Eberle, C., Palinski, W. and Ament, C. (2013). A novel mathematical model detecting early individual changes of insulin resistance. Diabetes Technology and Therapeutics, 15(10), 870-880. doi: $10.1089 /$ dia.2013.0084

[21] Ferrannini, E., Bjorkman, O., Reichard, G. A., Pilo, A., Olsson, M., Wahren, J. and De Fronzo, R. A. (1985). The disposal of an oral glucose load in healthy subjects: A quantitative study. Diabetes, 34(6), 580-588. doi: 10.2337/diabetes.34.6.580

[22] Garg, N., Thakur, S., McMahan, C. A. and Adamo, M. L. (2011). High fat diet induced insulin resistance and glucose intolerance are gender-specific in IGF-1R heterozygous mice. Biochemical and Biophysical Research Communications, 413(3), 476-480. doi: 10.1016/j.bbrc.2011.08.123

[23] Ha, J., Satin, L. S. and Sherman, A. s. (2016). A mathematical model of the pathogenesis, prevention and reversal of type 2 diabetes. Endocrinology, 157(2), 24-635. doi: 10.1210/en.2015-1564

[24] Hall, H., Perelman, D., Breschi, A., Limcaoco, P., Kellogg, R., McLaughlin, T. and Snyder, M. (2018). Glucotypes reveal new patterns of glucose dysregulation. PLOS Biology, 16(7), e2005143(1-23), doi: 10.1371/journal.pbio.2005143

[25] Harrell, C. S., Gillespie, C. F. and Neigh, G. N. (2016). Energetic stress: The reciprocal relationship between energy availability and the stress response. Physiology and Behavior, 166(1), 43-55. doi: $10.1016 / \mathrm{j}$.physbeh.2015.10.009

[26] Hendrix, E. M. T. and Tóth, B. G. (2010). Introduciton to nonlinear and global Optimization. New York: Springer. doi: 10.1007/978-0-387-88670-1

[27] Hezarkhani, S., Bonakdaran, S., Rajabian, R., Shahini, N. and Marjani, A. (2013).Comparison of glycemic excursion in patients with new onset type 2 diabetes mellitus before and after treatment with repaglinide. The Open Biochemistry Journal, 7, 19-23. doi: 10.2174/1874091x01307010019

[28] Hinson, J., Raven, P. and Chew, S. (2010). Insulin and the regulation of plasma glucose. In Hinson, J., Raven, P. and Chew, S. (Eds.), The Endocrine System (pp. 129-145). Elsevier. doi: 10.1016/b978-0-7020-3372-8.00011-2

[29] Hostalek, U. (2019). Global epidemiology of prediabetes - present and future perspectives. Clinical Diabetes and Endocrinology, 5(1), 1-5. doi: 10.1186/s40842-019-0080-0

[30] Kuo, S. C., Li, Y. and Cheng, J. T. (2018). Glucose tolerance test applied in screening of antidiabetic agent(s). qemphCurrent Research in Diabetes and Obesty Journal, 7(4), ID-555716. doi: 10.19080/crdoj.2018.07.555716

[31] Li. L., Li, X., Zhou, W. and Messina, J. L. (2013). Acute psychological stress results in the rapid development of insulin resistance. Journal of Endocrinology, 217(2), 175-184. doi: 10.1530/joe$12-0559$

[32] Ling. Z. and Pipeleers, D. G. (1996). Prolonged exposure of human beta cells to elevated glucose levels results in sustained cellular activation leading to a loss of glucose regulation. Journal of Clinical Investigation, 98(12), 2805-2812. doi: 10.1172/jci119108

[33] Maedler, K., Spinas, G. A., Lehman, R., Sergev, P., Weber, M., Fontana, A., Kaiser, N. and Donath, M. Y. (2001). Glucose induces-cell apoptosis via upregulation of the fas receptor in human islets. Diabetes, 50(8), 1683-1690. doi: 10.2337/diabetes.50.8.1683

[34] Marik, P. E. and Bellomo, R. (2013). Stress hyperglycemia: An essential survival response! Critical Care, 17(2), 305(1-7). doi: 10.1186/cc12514

[35] Melzer, k. (2011). Carbohydrate and fat utilization during rest and physical activity. The European e-Journal of Clinical Nutrition and Metabolism, 6(2), e45-52. doi: 10.1016/j.eclnm.2011.01.005

[36] Neil, W. P. and Hemmen, T. M. (2011). Neurologic manifestations of hypoglycemia. In Rigobelo, E. (Ed.), Diabetes - Damages and Treatments (pp. 259-274). InTech. doi: 10.5772/22204

[37] Nyarko, K. E. and Scitovski, R. (2004). Solving the parameter identification problem of mathematical model using genetic algorithms. Applied Mathematics and Computation, 153(3), 651 658. doi: 10.1016/s0096-3003(03)00661-1

[38] O'Keefe, J. H. and Bell, D. S. H. (2007). Postprandial hyperglycemia/hyperlipidemia (postprandial dysmetabolism) is a cardiovascular risk factor. The American Journal of Cardiology, 100(5), 899904. doi: 10.1016/j.amjcard.2007.03.107

[39] Paolisso, G., Rizzo, M. R., Barbieri, M., Manzella, D., Ragno, E. and Maugeri, D. (2003). Cardiovascular risk in type 2 diabetics and pharmacological regulation of mealtime glucose excursions. Diabetes and Metabolism, 29(4), 335-340. doi: 10.1016/s1262-3636(07)70044-7 
[40] Roepke, T. A., Yasrebi, A., Villalobos, A., Krumm, E. A., Yang, J. A. and Mamounis, K. J. (2017). Loss of ER $\alpha$ partially reverses the effects of maternal high-fat diet on energy homeostasis in female mice. Scientific Reports, 7, 6381(1-15). doi: 10.1038/s41598-017-06560-x

[41] Scitovski, R. and Jukić, D. (1996). A method for solving the parameter identification problem for ordinary differential equations of the second order. Applied Mathematics and Compution, 74(2-3), 273-291. doi: 10.1016/0096-3003(95)00098-4

[42] Service, F. J., Molnar, G. D., Rosevear, J. W., Ackerman, E., Gatewood, L. C. and Taylor, W. F. (1970). Mean amplitude of glycemic excursions, a measure of diabetic instability. Diabetes, 19(9), 644-655. doi: 10.2337/diab.19.9.644

[43] Shabestari, P. S., Panahi, S., Hatef, B., Jafari, S. and Sprott, J. C. (2018). A new chaotic model for glucose-insulin regulatory system. Chaos, Solitons and Fractals, 112, 44-51. doi: 10.1016/j.chaos.2018.04.029

[44] Suwannaphant, K. (2017). Association between socioeconomic status and diabetes mellitus: The national socioeconomics survey, 2010 and 2012. Journal of Clinical and Diagnostic Research, 11(7), LC18-LC22. doi: $10.7860 / j c d r / 2017 / 28221.10286$

[45] Tabák, A. G., Herder, C., Rathmann, W., Brunner, E. J. and Kivimäki, M. (2012). Prediabetes: a high-risk state for diabetes development. The Lancet, 379(9833), 2279-2290. doi: 10.1016/s01406736(12)60283-9

[46] Topp, B. Promislow, K., Devries, G., Miura, R. M. and Finegood, D. T. (2000). A model of $\beta$-cell mass, insulin and glucose kinetics: Pathways to diabetes. Journal of Theoretical Biology, 206(4), 605-619. doi: 10.1006/jtbi.2000.2150

[47] van der Kooij, M. A., Jene, T., Treccani, G., Miederer, I., Hasch, A., Voelxen, N., Walenta, S. and Müller, M. B. (2018). Chronic social stress-induced hyperglycemia in mice couples individual stress susceptibility to impaired spatial memory. Proceedings of the National Academy of Sciences, 115(43), E10187-E10196. doi: 10.1073/pnas.1804412115

[48] Wolfram Research, (2016). Mathematica, version 11.0. Champaign, Illinois, USA: Wolfram Research, Inc. https://www.wolfram.com 